\title{
The Multi-Faceted Challenges of Health Technology in Low - and Middle - Income Countries
}

\author{
Pamela Steele $^{1}$, Foyeke Tolani ${ }^{1}$ and Lakshmy Subramanian ${ }^{2 *}$ \\ ${ }^{1}$ Pamela Steele Associate Ltd, Prama House, Oxford, UK \\ ${ }^{2}$ Cranfield University, UK \\ *Corresponding Author: Lakshmy Subramanian, Cranfield University, UK.
}

Received: October 22, 2019; Published: October 23, 2019

DOI: $10.31080 /$ ASPS.2019.03.0426

\begin{abstract}
Technology has emerged as an important tool to improve health outcomes by promoting better access to healthcare and essential medicine. However, many people lack access to health technology due to various challenges faced by low- and middle-income countries (LMICs). The lack of technology can contribute to the deterioration of public health and increase the burden on alreadyconstrained health systems. This paper seeks to provide an overview of the mentioned challenges from a multi-disciplinary perspective. It shows that a combination of factors hinders health technology absorption and utilisation in LMICs, namely, poor resources, cultural influences, political motivations, inadequate existing healthcare structure, supply-side bottleneck, information gaps, among others. Addressing these hinderances through the suggested recommendations will lead to the improvement of health outcomes and the well-being of the population through health technology.
\end{abstract}

Keywords: Health Technology; Developing Countries; Challenges; Improved Health

\section{Background}

Health technologies as explained by the World Health Organization, is recognised as the application of organised knowledge and skills to solve health problems and improve the quality of life. This can be applied in the form of health equipment, medicines, vaccines, procedures, and other systems. Health technology provides advanced decision support, and when integrated with supporting technology, present opportunities for transforming healthcare transformation [1]. Health technologies can contribute to the reduction of human error, improving scientific outputs, accelerated medical coverage, and tracking of data for proactive healthcare policies [2]. It is an important tool in promoting better access to healthcare and essential medicines. However, many people in developing low- and middle-income countries (LMICs) lack access to even basic health technology and essential medicines, vaccines, and life-enhancing medicines. These deprivations have serious health consequences, especially in poor low-income countries, which are already strained by deteriorating health indicators [3].
In recent years, poor access to health commodities, including necessary technology, has been discussed on global platforms and policy agendas. This is because, soon, LMICs will have to find a sustainable way to procure health commodities and technology [4]. Also, evidence reveals that development assistance to LMICs should aim at broader healthcare system improvements, like human resources shortages, weak health technologies, logistic challenges, etc [5]. Access to health technology is not limited to technical and logistic challenges; it encompasses social values, economic interests, and political motivations, i.e., it is a continuous process involving the collaboration of different stakeholders.

Against this backdrop, the current study highlights the various challenges that LMICs experience in the process of integrating various health technologies in their existing health systems. It is important to identify these challenges and realise that they comprise a broad spectrum of issues, not limited to technical constraints. The study attempts to understand the various challenges through a re- 
view of the health technologies and the experiences of LMICs in using them.

\section{Barriers to health technology in LMICs}

This section seeks to identify problems particular to LMICs that need to be understood in the context of health technology. Although different kinds of technical interventions are taking place, the following challenges undermine their seamless integration in the current health systems.

Limited human resources: It is widely understood that the physical, human, and capital resources needed to synthesise technology into the health systems of LMICs are short in supply [6]. Inadequacies in human resources include not only the low density of medical professionals, but also an insufficient number of technical innovators, administrative staff, and equipment maintenance specialists. This is most critical at the primary care level, where the introduction of technology can result in considerable health gains [7]. The effectiveness of health technology largely depends on the foundations of the health sector, and its use will have financial and social implications for the country.

Morbidity patterns: A significant section of the population in LMICs is affected by health problems that have been eliminated or reduced to the minimum in most high-income countries [8]. That means that the application of health technologies in LMICs should be more problem-oriented, instead of replicating what is most frequently used. This situation becomes even more complex when the growth of LMICs is accompanied by an epidemiological transition, leading to the need for health technologies to adapt to the changing disease patterns [9].

Structure of the healthcare system: The incorporation and utilisation of health technologies will depend on the structure of the prevalent healthcare system in the country. Features that have a direct bearing on health technology are access, cost, human resources, and public-private partnerships, among others. The quality of care delivered is determined by its structure, processes, and outcome [10]; health technology is an essential element of the structure and process and can be critical in deciding the health outcome. The integration of technology has the potential to bring LMIC health outcomes closer to those of high-income countries. However, its introduction alone cannot achieve that; it should be approached with consideration for the country's contextual need and stage of economic development [11].
Culture: The trajectories of economic growth and social development have a very close link with the diverse cultures of LMICs. Culture influences the value system and, hence, can impact and be impacted by the use of technology. Likewise, culture affects the perception of health and related technologies. Low demand for health interventions often derives from attitudes deeply rooted in cultural and social norms, such as continued preferences for traditional over modern therapies. Likewise, gender is an important determinant of health-seeking behaviour. Raising access to maternal, reproductive, and child health is a major challenge within societies that restrict the public lives of women. There is evidence from Indonesia that the utilisation of prenatal care increases with the control a woman exercises over household finances $[12,13]$. Therefore, interventions to introduce technology must strive for acceptance. If the values incorporated by health technology conflict with the local values, leading to cultural imbalance, then the acceptance rates of the particular technology will be low [14]. Appropriate health technology based on low-cost medical care and that does not contradict existing cultural beliefs, is usually regarded to be more fruitful in LMICs. But this compatibility is often difficult to achieve until and unless the technology is an endogenous solution to a local health problem [15].

Political stewardship: In every country, public health policy is governed by the leaders and policymakers; in many, their leadership is not in line with internationally recognised measurements for the improvement of health outcomes. Managing medical innovations and related technologies can be a formidable task since it can involve a high degree of uncertainty. However, if health technologies are managed and monitored by the leaders, there is a stronger potential for sustainability. Therefore, it is imperative that all countries, especially LMICs, have guidelines, based on rigorous evidence, to inform practitioners, taxpayers, beneficiaries, and policymakers of which technologies work well, and which should be modified, or abandoned [16].

Information management: LMICs suffer limitations in the collection, assimilation, and analysis of data. The dissemination of the analysed data is also limited. Most LMICs have surveillance systems to track, collect, and monitor data regarding the use of different health technologies [17]. This helps in controlling the inherent risks of health technologies and improving the dissemination of the required information. Yet, initiatives to collect data at the health centre level and analyse it are poor, resulting in the understandable frustration and resistance against health technologies [18]. 
Capacity for technology absorption: Although health technology is being introduced, the question of appropriateness has arisen many times, and solutions on the appropriate technology and the right time to introduce them are being sourced [19]. Moreover, as many LMICs lack the physical and human infrastructure that must be in place to support and sustain the introduction of these technologies [20]. In the absence of such capacity, major challenges can arise in a country after the technology transfer takes place. Therefore, as already stated, health technology should be appropriate to the developing country's capabilities and factor endowments

Access to technology: Identification of the relevant health technology for an LMIC should not be viewed as the endpoint in the complicated process of improving access to technology. The technology needs to be managed throughout the process to address the numerous barriers along the pathway of the end-user [21]. Moreover, many of the health technology developers might not have experience of entering and operating in LMICs [22]. Therefore, improving access to health technologies in LMICs should be a collaborative effort.

User adoption of health technology: The adoption of health technology is influenced by the characteristics of the technology, the health issues it addresses, and the social, cultural, and political context. In LMICs, most imported technologies often lack the understanding of the needs of end-users and, hence, suffer from poor adoption rates [23].

Affordability: The cost of health technology is a major barrier, and LMICs find it difficult to embed technical knowledge. To address this, countries will have to adopt multiple strategies like bulk-purchasing, competitive bidding, tiered pricing, etc. LMICs rely on external funding, which does not guarantee long-term sustainability because donors' fatigue can limit the duration of the assistance [24].

Supply-side strategies: Suppliers of medical technologies often lack complete information about the demand in LMICs [25]. As a result, the supplier might not be aware of the absorptive capacity for such a technology in LMICs, and not take the necessary steps to enter the market. Likewise, the national governments do not have information on the demand for health technology either, due to weak quantification functions. Commercial partners who develop health technology for LMICs are also very rare. Reducing these information asymmetries by regularly updating and sharing the forecasts for medical technology can contribute to increased availability.

\section{Recommendations}

- $\quad$ Promote more cooperation and exchange of experiences among LMICs, as well as cooperation with experienced entities in high-income countries.

- Develop a greater sensitivity to the role that culture plays in determining the effectiveness of health technologies. This will help develop a pragmatic approach to the integration and utilisation of health technologies and improve its intended impacts.

- New technologies introduced in an LMIC should be assessed properly and considered as 'new' until they are completely disseminated, i.e., it reaches all the end-users who might need it. This will ensure the appropriate and suitable allocation of health technologies.

- Stronger contributions from technology champions will facilitate stronger impact. In global health, technology champions are individuals and organisations that advocate for new health technologies and are committed to improving access to them in LMICs [26]. These champions could also be technical agencies, research organisations, academic institutions, non-profit organisations, etc. Their role can include creating awareness about health technology and overcoming access barriers. They are responsible for identifying strategies to navigate through LMICs and establish the relationship among the different stakeholders.

- Adopt strategies to help developers understand users' perspectives, including through market research and surveillance, investigating consumer behaviour, applying research findings to product development and programme design, engaging in local capacity development, and creating strategies for national and local ownership in programme implementation.

- Develop strategies to guarantee the continuity of evaluation programmes and to reduce their sensitivity to structural changes.

- Development assistance should also be channelled into strengthening the health systems by improving the basic infrastructure, improving logistic capabilities, and empowering healthcare staff through training and professional development. 


\section{Conclusion}

The diffusion of health technology in developing countries faces numerous challenges. They are usually not caused due to a standalone reason but typically arise due to a combination of market failures, asymmetry of information, poor leadership, etc. The challenges can be different depending on the type of health technology. Still, these defeat the basic purpose of the health technology and reduce its impact. Addressing these bottlenecks requires united efforts at the global, national, and local level, as well as economic changes, cultural stimuli, political willingness, and a strong influence on people's perception of health and technology.

\section{Bibliography}

1. Thimbleby H. "Technology and the future of healthcare". Journal of Public Health Research 2 (2013): e28.

2. Patel VL and Kannampallil TG. "Human factors and health information technology: current challenges and future directions". Yearbook of Medical Informatics 9 (2014): 58-66.

3. Willems JS and Banta HD. "Improving the Use of Medical Technology”. Health Affairs 1.2 (1982).

4. Silverman R. "Projected Health Financing Transitions: Timeline and Magnitude- Working Paper 488". Center for Global Development (2017).

5. Michaud C. Development Assistance for Health: Recent Trends and Resource Allocation. Boston: Harvard Center for Population Development (2003).

6. Bleakley H. "Health, Human Capital, and Development". Annual Review of Economics 2 (2010): 283-310.

7. Alotaibi YK and Federico F. "The impact of health information technology on patient safety". Saudi Medical Journal 38.12 (2017): 1173-1180.

8. O'Donnell 0. "Access to health care in developing countries: breaking down demand side barriers". Cadernos de Saúde Pública 23 (2007): 2820-2834.

9. McKeown RE. "The Epidemiologic Transition: Changing Patterns of Mortality and Population Dynamics". American Journal of Lifestyle Medicine 3 (2009): 19S-26S.

10. Donabedian A. "Evaluating the Quality of Medical Care". The Milbank Quarterly 83 (2005): 691-729.
11. Perkins R and Neumayer E. "The International Diffusion of New Technologies: A Multi-Technology Analysis of Latecomer Advantage and Global Economic Integration". Annals of the Association of American Geographers 95 (2005): 789-808.

12. Beegle K., et al. "Bargaining power within couples and the use of prenatal and delivery care in Indonesia". Study Family Planning 32 (2001): 130-146.

13. Castro-Leal F., et al. "Public spending on health care in Africa: do the poor benefit?" World Health Organisation 78 (2000): 66-74.

14. Roundtable on the Promotion of Health Equity and the Elimination of Health Disparities; Board on Population Health and Public Health Practice; Institute of Medicine. Leveraging Culture to Address Health Inequalities: Examples from Native Communities: Workshop Summary. Washington (DC): National Academies Press (US); A, Culture as a Social Determinant of Health (2013).

15. Parker A., et al. "Appropriate tools for health care. Developing a technology for primary health care and rural development". WHO Chronicle 31 (1977): 131-137.

16. Carpenter DP. "The Political Economy of Food and Drug Agency Drug Review: Processing, Politics, and Lessons for Policy”. Health Affairs 23 (2004): 52-63.

17. Soto G., et al. "Challenges in the implementation of an electronic surveillance system in a resource-limited setting: Alerta, in Peru". BMC Proceedings 2 (2008): S4.

18. Koivusalo MT. "Finance, data and technology initiatives for noncommunicable disease control". Bulletin of the World Health Organization 97 (2019): 166-168.

19. WHO Technical Discussions. Appropriate Health Technology (1997).

20. Annies A. "Current Issues and Challenges in Managing Government's Assets and Facilities" (2007).

21. Juma C., et al. "Global Governance of Technology: Meeting the Needs of Developing Countries". International Journal of Technology Management 22 (2001): 629-655.

22. Program for Appropriate Technology in Health, Health Tech Historical Profile: Vaccine Vial Monitors (2005). 
23. James J. "The Diffusion of IT in the Historical Context of Innovations from Developed Countries". Social Indicators Research 111 (2013): 175-184.

24. Dieleman JL., et al. "Development assistance for health: past trends, associations, and the future of international financial flows for health". Lancet 387 (2016): 2536-2544.

25. Yadav P. "Health Product Supply Chains in Developing Countries: Diagnosis of the Root Causes of Underperformance and an Agenda for Reform". Health Systems and Reform 1.2 (2015): 142-154.

26. Free MJ. "Achieving appropriate design and widespread use of health care technologies in the developing world. Overcoming obstacles that impede the adaptation and diffusion of priority technologies for primary health care". International Journal of Gynaecology and Obstetrics 85 (2004): S3-S13.

Volume 3 Issue 11 November 2019

(C) All rights are reserved by Lakshmy Subramanian.,

et al. 